Muha, G. M. (1978) J. Magn. Reson. 32, 121-129.

Nilges, M. J. (1979) Ph.D. Thesis, University of Illinois.

Nishino, T., Tsushima, K., Hille, R., \& Massey, V. (1982)

J. Biol. Chem. 257, 7348-7353.

Olson, J. S., Ballou, D. P., Palmer, G., \& Massey, V. (1974)

J. Biol. Chem. 249, 4362-4382.

Patterson, D. B., Peterson, G. E., \& Carnevale, A. (1973)

Inorg. Chem. 12, 1282-1286.
Pick, F. M., McGartoll, M. A., \& Bray, R. C. (1971) Eur. J. Biochem. 18, 65.

Whitmore, F. C., \& Woodward, G. E. (1941) Organic Syntheses, Collect. Vol. I, p 159, Wiley, New York.

Williams, J. W., \& Bray, R. C. (1981) Biochem. J. 195, 753-760.

Williams, J. W., Morrison, J. F., \& Duggleby, R. G. (1979) Biochemistry 18, 2567-2573.

\title{
Rapid Kinetics of Agonist Binding and Permeability Response Analyzed in Parallel on Acetylcholine Receptor Rich Membranes from Torpedo marmorata $^{\dagger}$
}

Thierry Heidmann, Julius Bernhardt, Eberhard Neumann, and Jean-Pierre Changeux*

ABSTRACT: Excitable acetylcholine receptor rich membrane fragments from Torpedo marmorata have been used to measure, in parallel, (1) the permeability response to the fluorescent cholinergic agonist Dns- $\mathrm{C}_{6}-\mathrm{Cho}$ (in the $0.1 \mu \mathrm{M}$ to millimolar concentration range) characterized by both the initial rate of $\mathrm{Li}^{+}$transport and the rate of channel closure using the rapid-mixing quench-flow technique and (2) the kinetics of interaction of Dns- $\mathrm{C}_{6}-$ Cho with the acetylcholine receptor sites using the rapid-mixing stopped-flow technique. Analysis of the kinetics of Dns- $\mathrm{C}_{6}-\mathrm{Cho}$ binding in the millisecond to minute time scale leads to the identification of at least three conformational states of the acetylcholine receptor: a "low-affinity" one $(\sim 50 \mu \mathrm{M})$ that can be interconverted in the fraction of a second to a transient state of "intermediate affinity" $(\sim 1 \mu \mathrm{M})$, followed by the final stabilization, in the second to minute time range, of a state of "high affinity" $(\sim 3$ $n M)$. Comparison of Dns- $\mathrm{C}_{6}-$ Cho binding data with the permeability response to the same agonist demonstrates that the binding to the low-affinity conformation(s) of the acetylcholine receptor sites coincides with the triggering of the permeability increase- or "activation"-and the transitions to the intermediate- and high-affinity states with the two-step process of channel closing-or "desensitization". The data are interpreted in terms of a minimum four-state "allosteric" model for the acetylcholine receptor.

\& Sakmann, 1976). On the other hand, the desensitization reaction can be decomposed in, at least, two steps: a rapid one in the $100 \mathrm{~ms}$ to second time scale and a slow one in the range of seconds (Sakmann et al., 1980; Feltz \& Trautmann, 1982).

Current biochemical investigations have led to the unambiguous identification of the protein that binds the neurotransmitter, the $A c C h$-receptor ( $\mathrm{AcChR}$ ) sensu stricto, and its purification in milligram quantities has been achieved by starting with postsynaptic membrane fragments isolated from fish electric organs. Its functional reincorporation into artificial membranes or liposomes has further demonstrated that this single protein still mediates $\mathrm{AcCh}$-regulated ion translocation and thus comprises the associated ion channel [reviews in Changeux (1981), Popot (1983), and Anholt et al. (1983)]. The elementary AcChR "light form" is a heterologous pentameric protein made up of four distinct and strongly associated polypeptide chains $\alpha_{2} \beta \gamma \delta$ [reviews in Karlin (1980), Changeux (1981), and Conti-Tronconi \& Raftery (1982)]. Covalent (and noncovalent) analogues of $\mathrm{AcCh}$ and snake venom $\alpha$ toxins selectively label the $\alpha$ chains, which therefore carry at least part of the $\mathrm{AcCh}$ binding sites (two sites per receptor molecule) [references cited in Tzartos \& Changeux (1983)]. Potential markers of the ion channel such as the

\footnotetext{
${ }^{1}$ Abbreviations: $\mathrm{AcCh}$, acetylcholine; $\mathrm{AcChR}$, acetylcholine receptor;

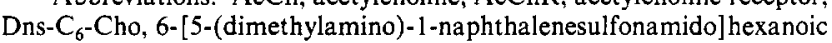
acid $\beta$-(methobromide) ethyl ester.
} 
noncompetitive blockers trimethisoquin or chlorpromazine block ion transport [see Adams (1981)] upon binding to either a unique high-affinity site on the $\mathrm{AcChR}$, which might be part of the ion channel, or a population of low-affinity "allosteric" sites [see Heidmann et al. (1982, 1983)]. They also label, upon UV irradiation, the $\delta$ chain and also, for some of them, the three other chains in a selective manner (Oswald \& Changeux 1981a,b; Heidmann et al., 1982, 1983). Functional and structural data thus suggest a strong coupling between the AcCh binding sites and the ion channel within the $\alpha_{2} \beta \gamma \delta$ oligomer.

Analysis of the kinetics of binding of $\mathrm{AcCh}$ and of the functional states of the ion channel has been carried out during the past few years by using a particularly convenient system on which both processes could be quantitatively studied. The AcChR-rich membrane fragments (Cohen et al., 1972; Duguid \& Raftery, 1973; Sobel et al., 1977) prepared from Torpedo electric organ make sealed vesicles or "microsacs", and the ion-permeability response of these microsacs can be measured as, for instance, the transport of radioactive cations (Kasai \& Changeux, 1971; Popot et al., 1976). Recent improvements in the microsac-isolation procedure (Hess et al., 1981; Bernhardt et al., 1981; Bernhardt \& Neumann, 1982) now provide a preparation containing a homogeneous population of wellsealed and functional vesicles, on which both the activation and the desensitization process can be evidenced [see also Popot et al. (1976)]. Finally, the recent introduction (Hess et al., 1979, 1981; Neubig \& Cohen, 1980; Neubig et al., 1982) of rapid-mixing quench-flow techniques permits the measurement of ion transport on the time scale of milliseconds [see also Moore \& Raftery (1980)], thus allowing a quantitative definition of the rate of agonist-triggered ion transport, a parameter proportional to the number of open channels, and of the rate and extent of desensitization [for theoretical treatment, see Bernhardt \& Neumann (1981, 1982)]. In agreement with the in vivo electrophysiological recordings, the desensitization of the permeability response appears as a two-step process (Walker et al., 1981, 1982; Hess et al., 1982).

In parallel, the kinetics of cholinergic-agonist binding were analyzed by a variety of techniques [review in Changeux (1981)]. Their effect on the kinetics of binding of radiolabeled snake $\alpha$ toxins provided the first evidence of a slow conformational transition resulting in the stabilization, at equilibrium, of a high-affinity state for agonists (Weber et al., 1975; Weiland et al., 1977). It then became evident that the AcChR might exist under several interconvertible conformations differing in ligand affinities (and possibly, also, in the functional state of the ion channel). Further quantitative definition of AcChR conformational equilibria resulted from the introduction of rapid-mixing techniques to measure the kinetics of binding of fluorescent (Heidmann \& Changeux, 1979) or radiolabeled (Boyd \& Cohen, 1980; Neubig et al., 1982) agonists. Two receptor conformations were clearly identified, and the affinity of the AcCh binding sites in both conformations (respectively $\sim 1 \mu \mathrm{M}$ and $\sim 3 \mathrm{nM}$ ), as well as the rate constants of the associated state transitions (second time range), was determined. Similar results were obtained by following the changes in the intrinsic fluorescence of the protein upon agonist addition (Barrantes, 1978). Finally, a third conformation of the AcChR was identified by following, in the millisecond time range and with a time resolution only accessible by spectroscopic techniques, the interaction of the receptor with the fluorescent agonist Dns- $\mathrm{C}_{6}$-Cho (Heidmann \& Changeux 1980). The equilibrium dissociation constant of Dns- $\mathrm{C}_{6}-$ Cho bound to this third conformation was found extremely high: in the $100 \mu \mathrm{M}$ range. This value is $\sim 100$-fold higher than that of the previously identified conformations of the receptor. It is in the range of the cleft concentration of AcCh during transmission of the nerve impulse and close to the apparent dissociation constant for activation by $\mathrm{AcCh}$ in vitro, suggesting that in this conformation of the AcChR the ionic channel would be open, whereas in the two other ones, of higher affinity, the channel would be shut. The data were interpreted in terms of a minimal four-state model (Neubig \& Cohen, 1980; Heidmann \& Changeux, 1980):

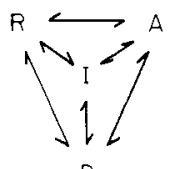

where A is the "active conformation", the only one with the ion channel open, $\mathrm{R}$ is the low-affinity, "resting", conformation susceptible to activation, and I and D are two close conformations of high affinity for agonists referred to as respectively "intermediate" and "desensitized".

In this paper, the permeability response of Torpedo marmorata microsacs is quantitatively analyzed by using the rapid-mixing quench-flow technique and compared with the rapid kinetics of Dns- $\mathrm{C}_{6}$-Cho binding analyzed in parallel on the same membrane fragments in the millisecond time scale.

\section{Materials and Methods}

Isolation of Sealed AcChR-Rich Microsacs. Sealed AcChR-rich membrane fragments were prepared from freshly dissected Torpedo marmorata electric organs following the procedure described by Bernhardt et al. (1981). Membranes were stored at $4{ }^{\circ} \mathrm{C}$ in Torpedo physiological saline solution (Na-Ringer, $250 \mathrm{mM} \mathrm{NaCl}, 5 \mathrm{mM} \mathrm{KCl}, 3 \mathrm{mM} \mathrm{CaCl}, 2 \mathrm{mM}$ $\mathrm{MgCl}_{2}, 5 \mathrm{mM}$ sodium phosphate, $\mathrm{pH} 7.0$ ) and used for ion-flux measurements and stopped-flow fluorescence experiments within 1-8 days. The concentration in $\alpha$-toxin binding sites was determined by centrifugation with ${ }^{3} \mathrm{H}$-labeled Naja nigricollis $\alpha$ toxin under conditions of at least 100-fold isotopic dilution [see Heidmann et al. (1983)].

Ion-Flux Assays. The AcChR-rich microsacs were first selectively filled with $\mathrm{Li}^{+}$ions following the method of Bernhardt et al. (1981). Routinely, $1 \mathrm{~mL}$ of concentrated membranes was diluted into $20 \mathrm{~mL}$ of $\mathrm{Li}^{+}-$Ringer (same as $\mathrm{Na}$ Ringer but with $\mathrm{NaCl}$ replaced by $\mathrm{LiCl}$ ), and $\mathrm{AcCh}$ was added as concentration "pulses" to the suspension under stirring (final instantaneous AcCh concentration at each pulse $0.1 \mathrm{mM}$ ), at 20 -s intervals ( $\sim 10$ pulses). Membranes were then pelleted by centrifugation for $30 \mathrm{~min}$ at $15000 \mathrm{rpm}$ in a Beckman rotor 30 , in the presence of a small amount of Percoll $(0.6 \% \mathrm{v} / \mathrm{v})$ to prevent stacking of the membranes at the bottom of the tube. After resuspension in $\mathrm{Na}$-Ringer, the membranes were passed through a Sephadex G-25 column in Na-Ringer to remove external $\mathrm{Li}^{+}$and residual amounts of $\mathrm{AcCh}$ and/or choline and diluted to a final concentration of $\sim 0.1 \mu \mathrm{M} \alpha$ toxin binding sites, in a $\mathrm{Na}$-Ringer solution supplemented with $0.1 \mathrm{mM}$ Tetram to inhibit acetylcholinesterase activity. $\mathrm{Li}^{+}$ content ranged from 150 to $300 \mathrm{~mol}$ of $\mathrm{Li}^{+} / \mathrm{mol}$ of $\alpha$ toxin binding site.

Quench-flow experiments were performed at $20^{\circ} \mathrm{C}$ in a Durrum D-133 multimixing device in both the continuous and interrupt modes. Accuracy of time calibrations was tested by monitoring alkaline hydrolysis of 2,4-dinitrophenyl acetate as described in Barman \& Gutfreund (1964). Quenching of $\mathrm{Li}^{+}$ efflux was achieved as in Neubig \& Cohen (1980) with a solution containing both a competitive antagonist and a channel 
blocker ( $4 \mathrm{mM} d$-tubocurarine and $0.4 \mathrm{mM}$ trimethisoquin). Vesicles were retained by manual filtration of the quenched membrane suspension on Millipore HAWP 025 filters washed 4 times with $5 \mathrm{~mL}$ of ice-cold $\mathrm{Na}$-Ringer, and their $\mathrm{Li}^{+}$ content was released by overnight incubation of the filters in counting vials containing $2.5 \mathrm{~mL}$ of $0.1 \%$ Triton $\mathrm{X}-100 ; \mathrm{Li}^{+}$ concentration in the vials was determined by flame-emission spectroscopy with an Instrument Laboratory 457 spectrophotometer.

The curves of $\mathrm{Li}^{+}$release established after mixing with the agonist were analyzed basically as described in Neubig \& Cohen (1980), in terms of two parameters: the initial ion flux, $k_{0}$, of $\mathrm{Li}^{+}$release and the rate constant, $k_{\mathrm{d}}$, for the time-dependent decline of $\mathrm{Li}^{+}$release referred to as "desensitization" of the permeability response. These parameters were determined by fitting the data to the equation

$$
X(t)=X_{\mathrm{ce}}+\left(X(0)-X_{\mathrm{ce}}\right) \exp \left[-\left(k_{0} / k_{\mathrm{d}}\right)\left[1-\exp \left(-k_{\mathrm{d}} t\right)\right]\right]
$$

where $X(t)$ is the fraction of $\mathrm{Li}^{+}$inside the vesicles at time $t$ and $X_{\text {ce }}$ is that after complete emptying of the excitable microsacs. This last value was determined by using a procedure developed by Bernhardt et al. (1981) in which microsacs retained on Millipore filters under negative pressure were submitted to repetitive sequential applications of $5 \mathrm{~mL}$ of $0.1 \mathrm{mM} \mathrm{AcCh}$ and $5 \mathrm{~mL}$ of $\mathrm{Na}$-Ringer.

Although undetectable in the second to minute time range, a slow reduction of the overall $\mathrm{Li}^{+}$content of the vesicles could be observed in the time course of hours; a leakage-flux correction was then made according to an exponential decay law, whose rate constant $\left(\sim 10^{-3} \mathrm{~min}^{-1}\right)$ was determined by systematically measuring $\mathrm{Li}^{+}$content at the beginning and at the end of the flux experiment. All the data presented in this paper are leakage-corrected values.

Stopped-Flow Fluorescence Experiments. Rapid kinetics of Dns- $\mathrm{C}_{6} \cdot$ Cho binding were followed at $20^{\circ} \mathrm{C}$ as previously described (Heidmann \& Changeux, 1979, 1980) with a Durrum stopped-flow apparatus equipped for fluorescence detection, on line with a Minc 23 Data General computer. Fluorescence signals were fitted by multiexponentials with a nonlinear iterative regression program as previously described (Heidmann \& Changeux, 1979).

Chemicals. Unlabeled and ${ }^{3} \mathrm{H}$-labeled $N$. nigricollis $\alpha$ toxins were a gift from Dr. A. Menez; trimethisoquin and Dns$\mathrm{C}_{6}$-Cho were a gift from Dr. G. Waksman, who synthesized them. Carbamoylcholine chloride, acetylcholine chloride, $d$-tubocurarine chloride, and Triton X-100 were from Sigma.

\section{Results}

Rapid Kinetics of Dns- $C_{6}$-Cho Binding. The conformational transitions of the membrane-bound AcChR were monitored by fluorescence stopped-flow techniques upon rapid mixing of the excitable microsacs with the fluorescent agonist Dns$\mathrm{C}_{6}$-Cho in the $0.1 \mu \mathrm{M}$ to millimolar concentration range [see Heidmann \& Changeux $(1979,1980)]$. The kinetic data were analyzed in terms of a four-state model (Neubig \& Cohen, 1980; Heidmann \& Changeux, 1980) involving (a) the transient population of an intermediate affinity state (I) and (b) the final stabilization of a high-affinity (D) conformation of the AcChR.

In the range of submicromolar Dns- $\mathrm{C}_{6}$-Cho concentration, the slow relaxation process recorded in the second to minute time range follows a single exponential time course (Figure 1). It corresponds to the equilibration of the AcChR toward the high-affinity D state (Heidmann \& Changeux, 1979; Boyd $\&$ Cohen, 1980). With increasing Dns- $\mathrm{C}_{6}$-Cho concentration,

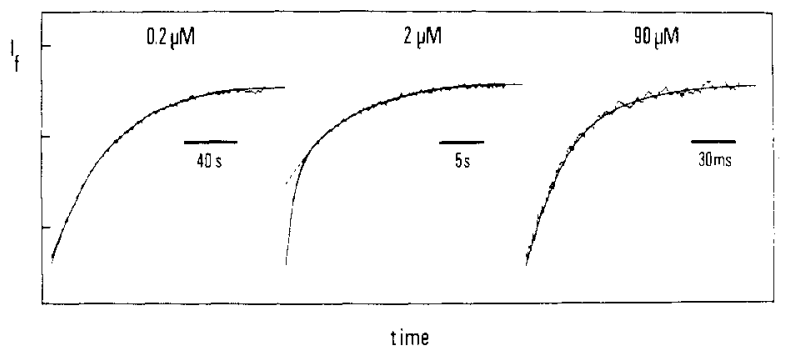

FIGURE 1: Rapid kinetics of Dns- $\mathrm{C}_{6}$-Cho interaction with membrane-bound $\mathrm{AcChR}$. Increases in fluorescence intensity after 1:1 rapid mixing in the stopped-flow apparatus of a suspension of AcChR-rich membrane fragments $(0.2 \mu \mathrm{M} \alpha$-toxin binding sites $)$ in Torpedo physiological solution with solutions of Dns- $\mathrm{C}_{6}$-Cho in the same medium (final concentrations after mixing indicated in the figure). The solid lines are the best fit of the traces by one (left and right) or two (middle) exponentials; the dotted line (middle) is the extrapolation to short times of the best single exponential fit to the last points. The three traces were recorded at the same gain, after adjustment of the base line.

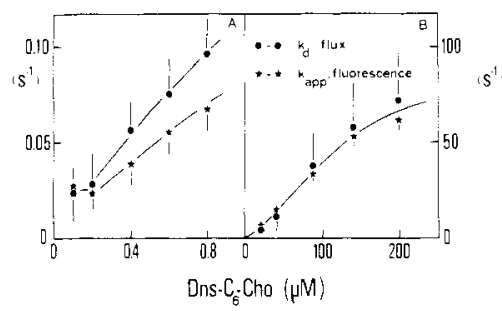

FIGURE 2: Rate constants characterizing channel closing and conformational transitions of AcChR. The rate constant of channel closing $\left(k_{\mathrm{d}}, 0\right)$ was derived as in Figure $4(\mathrm{~B})$ or $5(\mathrm{~A})$. The conformational transition of the AcChR toward the I (B) and D (A) states was monitored with Dns- $\mathrm{C}_{6}$-Cho in the corresponding domains of concentration, as in Figure 1, and the rates of isomerization $\left(k_{\mathrm{app}}\right.$, solid star) were derived by least-squares fit of the traces (correct fit by single exponentials in the two domains of Dns- $\mathrm{C}_{6}$-Cho concentrations of the figure, see Figure 1).

a second relaxation process referred to as "intermediate" is observed in the $10 \mathrm{~ms}$ to second time range (Figure 1). Its amplitude increases with increasing Dns- $\mathrm{C}_{6}{ }^{-}$- . to reach maximal amplitude at concentrations higher than $\sim 10 \mu \mathrm{M}$ (Figure 1). This intermediate relaxation process corresponds to the transient population of the intermediateaffinity state I (Heidmann \& Changeux, 1980), and the rate constants for this transition as well as those for the transition toward the D state are given in Figure 2.

From the variation of the apparent rate constant of the intermediate fluorescence relaxation process with agonist concentration the binding properties of the $\mathrm{AcChR}$ in the conformation existing prior to the state transition toward the I state can be inferred [see Heidmann \& Changeux (1980)]. A plot of $k_{\text {app }}$ as a function of Dns- $\mathrm{C}_{6}$-Cho concentration (Figure 3A) shows that $k_{\text {app }}$ increases in a sigmoid manner $\left(n_{\mathrm{H}}=1.9\right.$, see insert) and reaches a plateau value in the range of 0.2-0.5 mM Dns-C ${ }_{6}-$ Cho. A simple kinetic scheme involving the rapid and low-affinity binding of two agonist molecules to the AcChR (in the R and/or A state), followed by a rate-limiting isomerization toward the I state, fits the data. As illustrated in Figure 3, the experimental $k_{\text {app }}$ values can be adequately fitted by eq 2 [see Heidmann \& Changeux (1980)]:

$$
k_{\mathrm{app}}=k_{2}\left[a /\left(a+K_{\lambda}\right)\right]^{2}+k_{-2}
$$

where $k_{2}$ and $k_{-2}$ are the onward and backward isomerization rate constants for the transition of the AcChR toward the intermediate-affinity state of the $\mathrm{AcChR}$ and $K_{\lambda}$ is the equilibrium dissociation constant of the low-affinity binding 


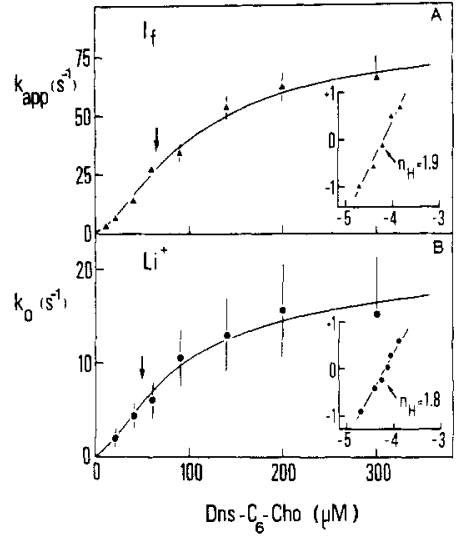

FIGURE 3: Compared variations of apparent rate constants of intermediate fluorescence relaxation process and of initial ion flux with Dns- $\mathrm{C}_{6}-$ Cho concentration. (A) The rate constant $k_{\mathrm{app}}$ of the intermediate fluorescence relaxation process (directly proportional to the fraction of liganded AcChR molecules in the R/A states, see text) was determined as in Figure 1. The solid line is the least-squares fit of the data to eq 2 and yields a dissociation constant for Dns- $\mathrm{C}_{6}-\mathrm{Cho}$ binding equal to $64 \pm 10 \mu \mathrm{M}$ (see arrow). (B) The initial ion flux $k_{0}$ (directly proportional to the fraction of opened channels) was determined by least-squares fit of the data as in Figure 4 to eq 1. The solid line is the least-squares fit of the data to eq 3 and yields an apparent dissociation constant equal to $48 \pm 10 \mu \mathrm{M}$ (see arrow). Inserts in both figures are Hill plots of the data with ordinates of log $\left(k / k_{\max }-k\right)$ and in the abscissas of $\log$ [Dns- $\mathrm{C}_{6}-\mathrm{Cho}$.

to the AcChR preceding the state transition in the I conformation. The best fit is obtained with $K_{\lambda}=64 \pm 10 \mu \mathrm{M}$.

Dns- $C_{6}$-Cho-Stimulated Ion Flux. The time courses of $\mathrm{Li}^{+}$ release from $\mathrm{AcChR}$-rich microsacs in the presence of various concentrations of Dns- $\mathrm{C}_{6}-$ Cho are presented in Figure 4. Qualitatively, it can be observed that (1) the initial flux of $\mathrm{Li}^{+}$ release significantly increases in the $100 \mu \mathrm{M}$ range of Dns$\mathrm{C}_{6}$-Cho concentrations and (2) the vesicles are not totally emptied even at long times after agonist addition. Similar conclusions hold for AcCh, although apparent quantitative differences are noticed. Quantitatively, the data are analyzed according to eq 1 (see Materials and Methods) to determine $k_{0}$, characterizing the initial flux of $\mathrm{Li}^{+}$release, and $k_{\mathrm{d}}$, the rate constant for desensitization of the permeability response, with the constraint of zero $\mathrm{Li}^{+}$released at zero time. The solid lines in Figure 4 represent the nonlinear least-squares fit of the experimental data, and the error bars in Figures 3 and 2 give the domains of confidence of the computer fit.

(A) Initial Ion Flux. The initial ion flux $k_{0}$ was derived for Dns- $\mathrm{C}_{6}$-Cho concentrations in the $10-300 \mu \mathrm{M}$ range, and the values obtained are plotted in Figure $3 \mathrm{~B}$ as a function of agonist concentration. As observed in the figure, $k_{0}$ first increases with increasing agonist concentration and levels off in the 150-200 $\mu \mathrm{M}$ range, half-maximum response being obtained at $\sim 90 \mu \mathrm{M}$ Dns- $\mathrm{C}_{6}-$ Cho. In addition, the curve has a sigmoid shape; a Hill plot of the data yields a Hill coefficient of $1.8 \pm 0.2$. If one assumes that two agonist molecules are required for channel opening, $k_{0}$ should vary as a function of Dns- $\mathrm{C}_{6}-$ Cho concentration according to the Hill equation:

$$
k_{0}=k_{0}^{\max }\left[a /\left(a+K_{\phi}\right)\right]^{2}
$$

where $a$ is the agonist concentration and $K_{\phi}$ is the apparent dissociation constant for activation. The least-squares fit of the data in Figure 3B (see solid line) yields a $K_{\phi}$ value equal to $48 \pm 10 \mu \mathrm{M}$.

(B) Desensitization of Permeability Response. Figure 4 shows that the permeability response desensitizes and leads to only partial emptying of the vesicles even long times after addition of the agonist. Desensitization develops and reaches
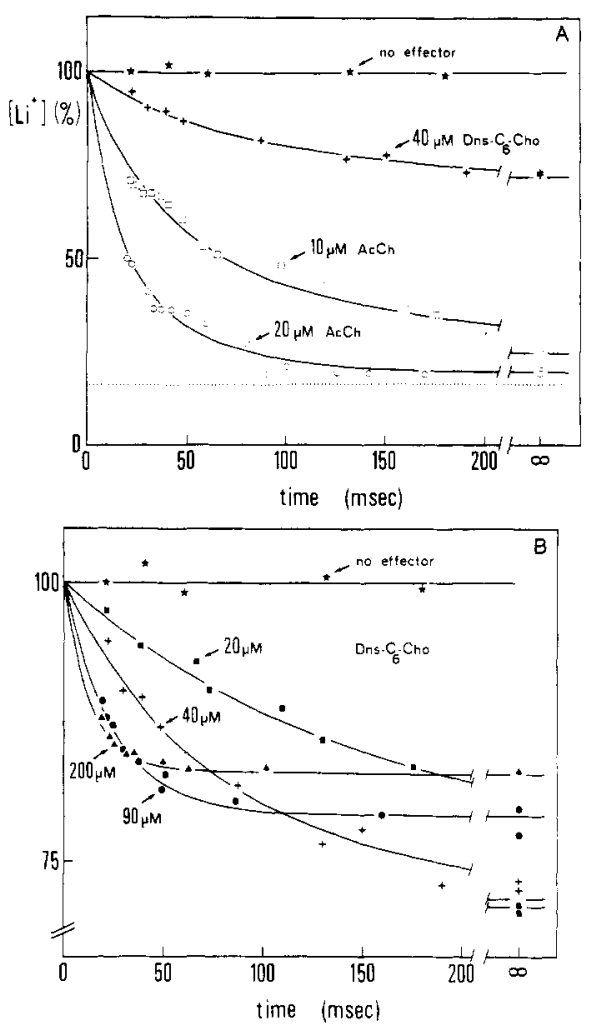

FIGURE 4: Quench-flow determination of agonist-triggered efflux of $\mathrm{Li}^{+}$from Torpedo microsacs. $\mathrm{Li}^{+}$-loaded AcChR-rich microsacs in Torpedo physiological solution (same conditions as in Figure 1) were rapidly mixed in a multimixing apparatus, first with a solution of $\mathrm{AcCh}$ [10 (D) or $20(0) \mu \mathrm{M}]$, or Dns-C 6 -Cho [20 (口), $40(+), 90(\bullet)$, or $200(\Delta) \mu \mathrm{M}$ ), or no effector (solid star), in the same medium and then, at the indicated times, with a quench solution (final concentration $2 \mathrm{mM}$ tubocurarine and $0.2 \mathrm{mM}$ trimethisoquin) to stop the agonist-stimulated efflux. $\mathrm{Li}^{+}$retained in the vesicles was determined by filtration and is expressed as a percentage of that in the absence of agonist. $\mathrm{Li}^{+}$content in the absence of agonist was $\sim 250 \mathrm{~mol}$ of $\mathrm{Li}^{+} / \mathrm{mol}$ of $\alpha$-toxin binding site. The dotted line in (A) indicates the $\mathrm{Li}^{+}$content of the vesicles after repetitive stimulation by $0.1 \mathrm{mM} \mathrm{AcCh}$ (complete emptying conditions, see text). Efflux curves for several Dns- $\mathrm{C}_{6}$-Cho concentrations are represented in (B) with an enlarged scale for the ordinates. The solid lines in both parts represent nonlinear least-squares fit of the data to eq 1 , including both activation and desensitization (see text). Data for 240-540-ms (omitted from the figure for clarity) and for $15-\mathrm{min}(\infty)$ efflux were included in the analysis. $k_{0}$ values for $\mathrm{AcCh}$ at 10 and $20 \mu \mathrm{M}$ were respectively 18 and $48 \mathrm{~s}^{-1}, k_{\mathrm{d}}$ values were respectively 8 and $14 \mathrm{~s}^{-1}$.

completion in the second or subsecond time range, depending on Dns-C 6 -Cho concentration. The values of $k_{\mathrm{d}}$ obtained by curve fitting of the data with eq 1 are given in Figure 2 for concentrations in the $10-300 \mu \mathrm{M}$ range.

At lower Dns- $\mathrm{C}_{6}$-Cho concentrations (submicromolar range), the amplitude of the flux response was too small to quantitate desensitization by the same curve-fitting procedure. A slightly different procedure was therefore used, on the basis of that developed by Hess and co-workers (Hess et al., 1981) with some modifications. Basically, desensitization was followed at given times after addition of Dns- $\mathrm{C}_{6}-\mathrm{Cho}$, by measuring the decrease of the initial flux of $\mathrm{Li}^{+}$release of the microsacs in response to a fixed concentation of carbamoylcholine. To carry out these measurements, the Durrum multimixer apparatus was used in a somewhat unconventional mode [see Guillain et al. (1981)]. As depicted in Figure 5, the microsacs were rapidly mixed with Dns- $\mathrm{C}_{6}-\mathrm{Cho}$, aged for given times in the delay tube, mixed in the second mixing chamber with a solution of carbamoylcholine, and finally quenched within the collection syringe. Under all conditions, the carbamoylcholine-triggered efflux took place between 


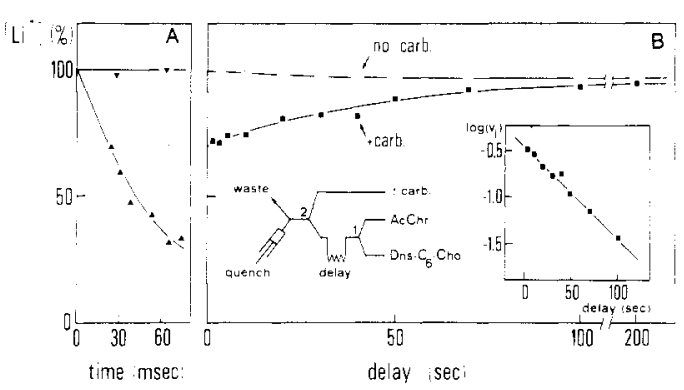

FIGURE 5: Decrease of ion-flux response to carbamoylcholine as a function of time of exposure of microsacs to low Dns- $\mathrm{C}_{6}$-Cho concentrations. (A) Time course of $\mathrm{Li}^{+}$efflux triggered by carbamoylcholine under identical conditions of concentration as in (B); the lower dotted line represents the $\mathrm{Li}^{+}$content of the vesicles under conditions of complete emptying (see Figure 4 and Materials and Methods). (B) The AcChR-rich membranes were incubated with Dns- $\mathrm{C}_{6}-\mathrm{Cho}$ in the delay tube for the periods of time indicated in the figure and rapidly mixed with carbamoylcholine. After $22 \mathrm{~ms}, \mathrm{Li}^{+}$ efflux $(\square)$ was quenched in the collection syringe (final volume $1 \mathrm{~mL}$ ), which already contained $250 \mu \mathrm{L}$ of quench solution (see the schematic diagram of the experiment in the figure). The flux response to Dns- $\mathrm{C}_{6}$-Cho alone ( $\square$ ) was measured as above but in the absence of carbamoylcholine. (Insert) Semilogarithmic plot of the decrease of the initial rate of $\mathrm{Li}^{+}$efflux (defined as $v_{\mathrm{i}}=\left(\mathrm{Li}^{+}{ }_{-}-\mathrm{Li}^{+}+{ }_{+}\right) /\left(\mathrm{Li}^{+}{ }_{-}^{-}\right.$ $\mathrm{Li}^{+}{ }_{c e}$ ), where $\mathrm{Li}^{+}$- and $\mathrm{Li}^{+}+$are respectively the $\mathrm{Li}^{+}$content in the absence and presence of carbamoylcholine (see above) and $\mathrm{Li}^{+}{ }_{\mathrm{ce}}$ is that after complete emptying [see (A)].

mixing chamber 2 and the entrance of the collection syringe, i.e., within a time $\theta$, which was taken minimal and was separately deduced from parallel acid-quench measurements of dinitrophenyl acetate hydrolysis $(\theta=22 \mathrm{~ms})$. A low carbamoylcholine concentration ( $0.4 \mathrm{mM}$ before quenching) was selected to keep the $\theta$-efflux values in the linear part of the carbamoylcholine-triggered efflux curve. It was taken as a reliable estimate of the fraction of activated receptors. In these conditions, it was observed (see Figure 5) that preincubation of the membranes with Dns- $\mathrm{C}_{6}$-Cho resulted in a time-dependent decrease of the initial flux of $\mathrm{Li}^{+}$release. A replot of the data in semilogarithmic coordinates (see insert) yielded the rate constant, $k_{\mathrm{d}}$, for this process (see values in Figure 2).

\section{Discussion}

We have used rapid-mixing techniques to measure under identical conditions and in parallel the kinetics of interaction of the fluorescent agonist Dns- $\mathrm{C}_{6}$-Cho with Torpedo microsacs and the kinetics of the agonist-triggered efflux of $\mathrm{Li}^{+}$from the same microsacs.

State Transitions of AcChR and Desensitization. As previously reported (Heidmann \& Changeux, 1979, 1980), two major state transitions of the $\mathrm{AcChR}$ are observed upon addition of Dns- $\mathrm{C}_{6}-\mathrm{Cho}$, one leading to the transient population of the intermediate (I) state-following the intermediate relaxation process-and the other to the final stabilization of the high-affinity (D) state-following the slow relaxation process. At high Dns- $\mathrm{C}_{6}$-Cho concentrations $(>10 \mu \mathrm{M})$, almost all the $\mathrm{AcChR}$ are rapidly interconverted to the I state, resulting in an almost zero amplitude of the slow relaxation process. On the other hand, at low Dns- $\mathrm{C}_{6}$-Cho concentrations, the AcChR molecules are directly interconverted to the D state without any significant population of the I state, as evidenced by the almost zero amplitude of the intermediate relaxation process. In these two extreme situations, the kinetics of $\mathrm{Li}^{+}$ efflux could be quantitatively determined. In the high Dns$\mathrm{C}_{6}$-Cho concentration range, the analysis of the observed response provided directly the rate constant $k_{\mathrm{d}}$ characterizing the desensitization of the response. In the low Dns- $\mathrm{C}_{6}-\mathrm{Cho}$ concentration range, because of the limited amplitude of the flux response, $k_{\mathrm{d}}$ was derived more indirectly by measuring the reduction of the initial flux of $\mathrm{Li}^{+}$release triggered by carbamoylcholine addition, after preincubation of the vesicles for various periods of time with Dns- $\mathrm{C}_{6}-\mathrm{Cho}$. Comparison of both sets of data in the appropriate domains of Dns- $\mathrm{C}_{6}$ - Cho concentrations (Figure 2) clearly reveals that the rate of desensitization of the permeability response, $k_{\mathrm{d}}$, is, within a factor of 2, identical with the rate of isomerization of the AcChR to the I and D states. It should therefore be concluded that both the I and D states correspond to desensitized conformations of the AcChR where the ion channel is closed. This conclusion confirms and further extends to the I state that derived in the case of the D state by Neubig et al. (1982). These authors followed in parallel the slow stabilization of the high-affinity state of the AcChR (using radiolabeled $\mathrm{AcCh}$ ) and the slow desensitization of the permeability response (following the rate of ${ }^{22} \mathrm{Na}$ transport). They found closely related values for both processes [see also Sine \& Taylor (1979)]. It is also noteworthy that Neubig \& Cohen (1980), on the basis of an analysis of rapid ion-flux measurements and available rapid binding data, predicted the existence of a state equivalent to the I state. The present analysis fully substantiates this prediction.

The demonstration, presented in this paper, that two distinct AcChR conformations are associated with closed channels can be correlated with the biphasic process of desensitization observed in vivo by electrophysiological techniques following either single-channel kinetics (Sakmann et al., 1980) or the decay of the end-plate conductance after ionophoretic application of agonists (Feltz \& Trautmann, 1982). In both cases, desensitization was a two-step process, and the values reported for the rate constant of the two steps (respectively 0.2 and 7 $\mathrm{s}^{-1}$ in the presence of $20 \mu \mathrm{M} \mathrm{AcCh}$ ) fall in the range of those measured with Dns- $\mathrm{C}_{6}$-Cho.

Also, it was shown (Walker et al., 1981, 1982; Hess et al., 1982) that, in vitro, the agonist-triggered ${ }^{86} \mathrm{Rb}$ entry into native or reconstituted vesicles declines with a biphasic time course, with maximum rates of, respectively, $\sim 0.07 \mathrm{~s}^{-1}$ and $\sim 2 \mathrm{~s}^{-1}$. These values are relatively low as compared to those predicted by Neubig et al. (1982) and those determined in the present study. Such differences might result, for instance, from differences in temperature $\left(0-4{ }^{\circ} \mathrm{C}\right.$ instead of $\left.20^{\circ} \mathrm{C}\right)$ between the two series of experiments. More likely, they originate from a systematic underestimation of the correct rates of desensitization, since the amount of $\mathrm{AcChR}$ accessible for activation was characterized by these authors by the response amplitude to high concentrations of agonist, under conditions where the response was not strictly proportional to the initial ion flux, and then to the number of opened channels. Finally, although in the present study a detailed analysis of the permeability response to $\mathrm{AcCh}$ was not presented, $k_{\mathrm{d}}$ values for $\mathrm{AcCh}$ were in the same range as those found for Dns- $\mathrm{C}_{6}-\mathrm{Cho}$ (see legend to Figure 4).

Low-Affinity Binding and Initial Ion Flux. One of the most striking results presented in this paper is the parallel rapid measurement of the low-affinity binding of Dns- $\mathrm{C}_{6}$-Cho to the $\mathrm{AcChR}$ [in the $\mathrm{R}$ and/or A state in Heidmann \& Changeux $(1979,1980)$, the " $R_{c}$ " and/or " $R_{0}$ " state in Neubig \& Cohen $(1980)$, the postulated "very low affinity state" in Walker et al. (1982)] and the rapid titration of the number of open channels, immediately after mixing with agonist. Under the present conditions, the initial transport rate $k_{0}$ is directly proportional to the number of receptors in the A state, and the rate constant $k_{\text {app }}$ of the intermediate fluorescence relaxation process is directly proportional to the fraction of liganded 
AcChRs. In both cases, the corresponding parameters were measured up to saturation (in the $300 \mu \mathrm{M}$ range), thus yielding complete concentration-effect curves. Their resemblance (see Figure 3) is remarkable. Both are sigmoid in shape with almost identical Hill coefficients and closely related apparent dissociation constants: in the $100 \mu \mathrm{M}$ range of Dns- $\mathrm{C}_{6}-\mathrm{Cho}_{0}$ concentration.

Similar sigmoid dependence of the number of open channels with agonist concentration has been observed in vivo by electrophysiological techniques [reviews in Steinbach (1980) and Adams (1981)] and also in vitro with initial rate measurements of ion fluxes in Electrophorus vesicles (Cash \& Hess, 1980), native or reconstituted Torpedo membranes (Neubig \& Cohen, 1980; Wu et al., 1981; Walker et al., 1982), and muscle cells in culture (Sine \& Taylor, 1980). It can be accounted for by the cooperative binding of at least two agonist molecules per molecule of AcChR (see Figure 3). The known structural and biochemical properties of the AcChR are consistent with this hypothesis [review in Changeux (1981)]. In addition, the apparent dissociation constant found here for channel activation by Dns- $\mathrm{C}_{6}$-Cho is similar to that characterizing the ${ }^{86} \mathrm{Rb}$ flux response of eel vesicles to $\mathrm{AcCh}\left[K_{\phi} \sim\right.$ $80 \mu \mathrm{M}$ (Hess et al., 1981; Cash et al., 1981)], that of ${ }^{22} \mathrm{Na}$ flux with Torpedo vesicles $\left[K_{\phi} \sim 70 \mu \mathrm{M}\right.$ (Neubig et al., 1982); $K_{\phi} \sim 45 \mu \mathrm{M}$ (Dunn \& Raftery, 1982)], or muscle cells in culture $\left[K_{\phi} \sim 40-50 \mu \mathrm{M}\right.$ (Catterall, 1975)]. Voltage-clamp studies with Electrophorus electrocyte (Sheridan \& Lester, 1977) or the frog neuromuscular junction (Dionne et al., 1978; Adams, 1981) also yielded similar values for the apparent dissociation constant for the permeability response to $\mathrm{AcCh}$ $\left(K_{\phi} \sim 25 \mu \mathrm{M}\right)$. In summary then, the rapid kinetic analysis of Dns- $\mathrm{C}_{6}-\mathrm{Cho}$ binding, in agreement with the available data from other laboratories, strongly supports the conclusion that activation is associated with a low-affinity interaction of two agonist molecules per molecule of AcChR.

The main difference found between the effects of Dns$\mathrm{C}_{6}$-Cho and $\mathrm{AcCh}$ is the relatively low values of the flux of $\mathrm{Li}^{+}$release, $k_{0}$, which are at least 20 -fold lower than those measured with AcCh (see Figure 4). A similar difference was observed by Neubig \& Cohen (1980) and Dunn \& Raftery (1982) in the case of the agonist phenyltrimethylammonium, for which the maximum ion flux was less than $10 \%$ of that found for carbamoylcholine.

A favorable consequence of the low $k_{0}$ values obtained with Dns- $\mathrm{C}_{6}-\mathrm{Cho}$ is that the rapid desensitization process could be easily detected. It manifested itself not only by a deviation of the efflux curves from single exponential but also by an important reduction of the equilibrium flux amplitude (see eq 1 with $k_{0}$ close to $k_{\mathrm{d}}$ ). In turn, the rapid desensitization might have been underestimated in the analysis of the kinetics of ion flux measured with other agonists, since they were systematically forced to fit with single-exponential processes (Neubig et al., 1982; Dunn \& Raftery, 1982; Walker et al., 1982).

At least two possible and nonexclusive explanations for the low $k_{0}$ values found with Dns- $\mathrm{C}_{6}-\mathrm{Ch}_{0}$ appear plausible within the framework of the adapted four-state allosteric model: (1) Dns- $\mathrm{C}_{6}-$ Cho binds in a nonexclusive (Rubin \& Changeux, 1966) manner to the open (A) state and to the resting (R) state. If one assumes the kinetic scheme

$$
\mathrm{R} \stackrel{K_{\mathrm{R}} / 2}{\longrightarrow} \mathrm{RL} \stackrel{2 K_{\mathrm{R}}}{\longrightarrow} \mathrm{RL}_{2} \stackrel{\mathrm{M}}{\rightleftharpoons} \mathrm{Al}_{2}
$$

where $\mathrm{R}$ is associated with a close channel conformation and A with an open channel one, then the isomerization constant, $M$, between the liganded close and open conformations of the AcChR would become much lower for this agonist than for carbamoylcholine assumed to bind exclusively to the A state. (2) Dns- $\mathrm{C}_{6}-$ Cho interacts with allosteric sites distinct from the agonist binding site, where it causes a reduction of the initial flux of $\mathrm{Li}^{+}$release.

Hypothesis 1 is consistent with data available on classical allosteric proteins [see, for instance, Changeux \& Rubin (1968) in the case of aspartate transcarbamylase]. They indicate that both the extent and rate of the conformational changes associated with ligand binding may differ from one ligand to another and result from the "partial" shift of the conformational equilibrium in favor of the active state. It is also consistent with the in vivo kinetic measurements on channel opening and closing, which strongly suggest that the maximum fraction of channels opened varies from one agonist to the other, with, for instance, twice as many channels opened with acetylcholine as compared with carbamoylcholine [see Adams (1981)]. According to this interpretation, the apparent dissociation constants measured for channel opening $\left(K_{\phi}\right)$ and low-affinity binding $\left(K_{\lambda}\right)$ should then be identical, as observed, and simply equal to $K_{\mathrm{R}}$ in eq 4 .

Hypothesis 1, however, does not simply account for the observed reduction of the maximum flux-response amplitude observed at high Dns- $\mathrm{C}_{6}$-Cho concentrations [see Figure 4 and Lindstrom et al. (1980) with carbamoylcholine]. Such effect, which develops at concentrations where already a large fraction of the channels are opened, indeed necessarily involves the interaction of the agonist with a second class of sites distinct from the $\mathrm{AcCh}$ binding sites. A similar conclusion was derived from the analysis of the rate of the intermediate relaxation process, which slightly decreases in the high (millimolar) Dns- $\mathrm{C}_{6}$-Cho concentration range [see Heidmann \& Changeux (1980)]. The exact nature of this (these) allosteric site(s) is still under investigation, as well as the mechanism by which they cause a reduction of ion flux. One possible interpretation [see also Adams (1981) and Walker et al. (1982)] could be that Dns- $\mathrm{C}_{6}-\mathrm{Ch}$, like several other agonists such as decamethonium [see Adams \& Sakmann (1978)] causes both channel opening and channel blockade. According to the available in vivo data on the rate of channel blocking [see Adams (1981)], this blocking effect would take place, with Dns- $\mathrm{C}_{6}-\mathrm{Cho}$ in the high concentration range, within a fraction of a millisecond. Therefore, it would not be kinetically resolved by the quench-flow technique and would simply result in an instantaneous decrease in the number of opened conducting channels. This was indeed observed in vitro with the noncompetitive channel-blocker procaine (Karpen et al., 1982), which at a concentration of $100 \mu \mathrm{M}$ actually reduced by a factor of 2 the initial rate of ion flux. This possibility is further strengthened by the evidence that Dns- $\mathrm{C}_{6}-$ Cho in its high concentration range can displace the noncompetitive blocker $\left[{ }^{3} \mathrm{H}\right]$ phencyclidine from its unique high-affinity site (Heidmann et al., 1983), with an apparent dissociation constant measured under equilibrium conditions equal to be $\sim 140 \mu \mathrm{M}$ (T. Heidmann, unpublished results). Accordingly, the $k_{0}$ values in Figure 3 at high Dns- $\mathrm{C}_{6}$-Cho concentrations would be underestimated, as well as, therefore, the value $K_{\phi}$ for channel opening and receptor activation (which would then be even closer to the $K_{\lambda}$ value derived from the fluorescence stopped-flow measurements).

In conclusion, although some deviations are observed in the high agonist concentration range, the ability to measure in parallel Dns- $\mathrm{C}_{6}-\mathrm{Cho}$ binding and the permeability response to this agonist under identical conditions allows the identification of the binding process associated with receptor activation and of the state transitions associated with rapid and slow 
desensitization. The data can be reasonably well fitted in terms of a four-state model of the AcChR [see Neubig \& Cohen (1980), Heidmann \& Changeux (1980), and the introduction]. Efficient channel activation occurs for high concentrations of Dns- $\mathrm{C}_{6}-\mathrm{Cho}$, leading to fully occupied biliganded $\mathrm{R} / \mathrm{A}$ receptors, and desensitization is associated with the transient population of the I conformation followed by the final equilibration of the $\mathrm{D}$ conformation of highest affinity.

In this model, the two AcCh binding sites have distinct affinities depending on the conformation of the AcChR, ranging from $\sim 50 \mu \mathrm{M}$ for the $\mathrm{R}$ conformation (see above), to $\sim 1 \mu \mathrm{M}$ for the I conformation [see Heidmann \& Changeux (1979)], and up to $\sim 3 \mathrm{nM}$ for the D state [see Heidmann \& Changeux (1979)]. This model differs from more complex and rather arbitrary schemes recently proposed (Dunn \& Raftery, 1982) where activation and desensitization have been assumed to proceed via distinct sites on the $\mathrm{AcChR}$ molecule.

\section{Acknowledgments}

We thank particularly Dr. G. Dagher and R. Garay (Prof. Meyer Unité INSERM U7, hopital Necker, Paris) for making their flame-emission spectrophotometer available to us, Prof. J. Yon and Dr. C. Ghelis (Laboratoire d'Enzymologie Physicochimique et Moléculaire, Orsay) for the use of their Durrum multimixing system in preliminary tests, and Dr. P. Champeil for helpful suggestions in the use of this equipment. We also acknowledge Dr. G. Waksman and Prof. B. Roques for the gift of Dns-C ${ }_{6}-$ Cho and Dr. A. Menez for the gift of $N$. nigricollis $\alpha$ toxin.

Registry No. Dns- $\mathrm{C}_{6}$-Cho, 60837-35-6; Li, 7439-93-2.

\section{References}

Adams, P. R. (1981) J. Membr. Biol. 58, 161-174.

Adams, P. R., \& Sakmann, B. (1978) Proc. Natl. Acad. Sci. U.S.A. 75, 2994-2998.

Anholt, R., Lindstrom, J., \& Montal, M. (1983) in Enzymes of Biological Membranes (Martonosi, A., Ed.) 2nd ed., Plenum Press, New York (in press).

Barman, T. E., \& Gutfreund, H. (1964) in Rapid-Mixing and Sampling Techniques in Biochemistry (Chance, B., Eisenhardt, R. H., Gibson, Q. H., \& Lonberg-Holm, K. K., Eds.) pp 339-343, Academic Press, New York.

Barrantes, F. J. (1978) J. Mol. Biol. 124, 1-26.

Bernhardt, J., \& Neumann, E. (1981) Biophys. Chem. 14, 303-316.

Bernhardt, J., \& Neumann, E. (1982) Biophys. Chem. 15, 317-341.

Bernhardt, J., Moss, K. M., Luckinger, R. M., \& Neumann, E. (1981) FEBS Lett. 134, 245-248.

Boyd, N. D., \& Cohen, J. B. (1980) Biochemistry 19, 5344-5358.

Cash, D. H., \& Hess, G. P. (1980) Proc. Natl. Acad. Sci. U.S.A. 77, 842-846.

Cash, D. J., Aoshima, H., \& Hess, G. P. (1981) Proc. Natl. Acad. Sci. U.S.A. 78, 3318-3322.

Catterall, W. A. (1975) J. Biol. Chem. 250, 1776-1781.

Changeux, J.-P. (1981) Harvey Lect. 75, 85-254.

Changeux, J.-P., \& Rubin, M. (1968) Biochemistry 7, 553-561.

Cohen, J. B., Weber, M., Huchet, M., \& Changeux, J.-P. (1972) FEBS Lett. 26, 43-47.

Conti-Tronconi, B. M., \& Raftery, M. A. (1982) Annu. Rev. Biochem. 51, 491-530.

Dionne, V. E., Steinbach, J. H., \& Stevens, C. F. (1978) J. Physiol. (London) 281, 421-444.
Duguid, J. R., \& Raftery, M. A. (1973) Biochemistry 12, 3593-3597.

Dunn, S. M. J., \& Raftery, M. A. (1982) Biochemistry 21, 6264-6272.

Feltz, A., \& Trautmann, A. (1982) J. Physiol. (London) 322, 257-272.

Guillain, F., Champeil, P., Lacapere, J. J., \& Gingold, M. P. (1981) J. Biol. Chem. 256, 6140-6147.

Heidmann, T., \& Changeux, J.-P. (1979) Eur. J. Biochem. 94, 255-279.

Heidmann, T., \& Changeux, J.-P. (1980) Biochem. Biophys. Res. Commun. 97, 889-896.

Heidmann, T., Oswald, R. E., \& Changeux, J.-P. (1982) C. R. Hebd. Seances Acad. Sci., Ser. D 295, 345-349.

Heidmann, T., Oswald, R. E., \& Changeux, J.-P. (1983) Biochemistry 22, 3112-3127.

Hess, G. P., Cash, D. J., \& Aoshima, H. (1979) Nature (London) 282, 329-331.

Hess, G. P., Aoshima, H., Cash, D. J., \& Lenchitz, B. (1981) Proc. Natl. Acad. Sci. U.S.A. 78, 1361-1365.

Hess, G. P., Pasquale, E. B., Walker, J. W., \& Mc Namee, M. G. (1982) Proc. Natl. Acad. Sci. U.S.A. 79, 963-967.

Karlin, A. (1980) in Cell Surface and Neuronal Function (Poste, G., Ed.) pp 191-260, Elsevier/North-Holland, New York.

Karpen, J. W., Aoshima, H., Abood, L. G., \& Hess, G. P. (1982) Proc. Natl. Acad. Sci. U.S.A. 79, 2509-2513.

Kasai, M., \& Changeux, J.-P. (1971) J. Membr. Biol. 6, 1-80.

Katz, B. (1966) in Nerve, Muscle \& Synapse, McGraw-Hill, New York.

Katz, B., \& Miledi, R. (1970) Nature (London) 226, 962-963.

Lindstrom, J., Anholt, R., Einarson, B., Engel, A., Osame, M., \& Montal, M. (1980) J. Biol. Chem. 255, 8340-8350.

Moore, H. P. H., \& Raftery, M. A. (1980) Proc. Natl. Acad. Sci. U.S.A. 77, 4509-4513.

Neher, E., \& Sakmann, B. (1976) Nature (London) 260, 799-802.

Neher, E., Marty, A., \& Fenwick, E. (1983) Prog. Brain Res. 58, 39-48.

Neubig, R. R., \& Cohen, J. B. (1980) Biochemistry 19, 2770-2779.

Neubig, R. R., Boyd, N. D., \& Cohen, J. B. (1982) Biochemistry 21, 3460-3467.

Oswald, R. E., \& Changeux, J.-P. (1981a) Biochemistry 20, 7166-7174.

Oswald, R. E., \& Changeux, J.-P. (1981b) Proc. Natl. Acad. Sci. U.S.A. 78, 3925-3929.

Popot, J. L. (1983) in Basic Mechanisms of Neuronal Hyperexcitability, pp 137-170, Alan R. Liss, Inc., New York.

Popot, J. L., Sugiyama, H., \& Changeux, J.-P. (1976) J. Mol. Biol. 106, 469-483.

Rubin, M., \& Changeux, J.-P. (1966) J. Mol. Biol. 21, 265-274.

Sakmann, B., Patlak, J., \& Neher, E. (1980) Nature (London) 286, 71-73.

Sheridan, R. E., \& Lester, H. A. (1977) J. Gen. Physiol. 70, 187-219.

Sine, S. M., \& Taylor, P. (1979) J. Biol. Chem. 254, 3315-3325.

Sine, S. M., \& Taylor, P. (1980) J. Biol. Chem. 255, 10144-10156.

Sobel, A., Weber, M., \& Changeux, J.-P. (1977) Eur. J. Biochem. 80, 215-224.

Steinbach, J. H. (1980) Cell Surf. Rev. 6, 191-260. 
Tzartos, S., \& Changeux, J.-P. (1983) EMBO J. 2, 381-387. Walker, J. W., Mc Namee, M. G., Pasquale, E., Cash, D. J., \& Hess, G. P. (1981) Biochem. Biophys. Res. Commun. $100,86-90$.

Walker, J. W., Takeyasu, K., \& Mc Namee, M. G. (1982) Biochemistry 21, 5384-5389.
Weber, M., David-Pfeuty, T., \& Changeux, J.-P. (1975) Proc. Natl. Acad. Sci. U.S.A. 72, 3443-3447.

Weiland, G., Georgia, B., Lappi, S., Chignell, C. E., \& Taylor, P. (1977) J. Biol. Chem. 252, 7648-7656.

Wu, W. C. S., Moore, H. H. P., \& Raftery, M. A. (1981) Proc. Natl. Acad. Sci. U.S.A. 78, 775-779.

\title{
Hydrogen Ion Cotransport by the Renal Brush Border Glutamate Transporter ${ }^{\dagger}$
}

\author{
Pamlea J. Nelson, Gary E. Dean, Peter S. Aronson, and Gary Rudnick*
}

ABSTRACT: Sodium ion dependent glutamate transport into rabbit renal brush border membrane vesicles is stimulated by low external $\mathrm{pH}$ and inhibited by low internal $\mathrm{pH}$. Imposition of a $\mathrm{pH}$ difference $(\Delta \mathrm{pH})$ (interior alkaline) across the vesicle membrane drives glutamate accumulation in the absence of other driving forces. This process requires $\mathrm{Na}^{+}$but is not due to generation of an $\mathrm{Na}^{+}$gradient in response to $\Delta \mathrm{pH}$. Internal $\mathrm{K}^{+}$stimulates both the rate and extent of glutamate accu-

The dicarboxylic amino acid glutamate is the substrate for specific transport systems in kidney and brain. In brain, the action of glutamate as a neurotransmitter is believed to be terminated by rapid and efficient reuptake into presynaptic nerve terminals (Curtis et al., 1960). Brain slices and synaptosomes accumulate glutamate by a high-affinity process (Navon \& Lathja, 1969; Balcar \& Johnson, 1972; Logan \& Snyder, 1972; Wheeler \& Hollingsworth, 1978) that has also been studied in cultured cerebellar cells (Stallcup et al., 1979) and in isolated synaptic plasma membrane vesicles (Kanner \& Sharon, 1978; Kanner \& Marva, 1982). In the kidney, glutamate (like other amino acids) is freely filtered and extensively reabsorbed in the proximal convoluted tubule (Weber, 1962). Glutamate is rapidly transported into intact proximal tubule epithelial cells (Siebernagl et al., 1975; Samarzija \& Fromter, 1975) and into renal brush border membrane vesicles (Weiss et al., 1978; Schneider et al., 1980; Burckhardt et al., 1980)

In many ways, glutamate transport is similar in brain and kidney. In both cases, glutamate influx involves $\mathrm{Na}^{+}$cotransport and $\mathrm{K}^{+}$countertransport (Schneider \& Sacktor, 1980; Kanner \& Sharon, 1978), the $K_{\mathrm{M}}$ for transport is in the micromolar range, and aspartate is a potent competitive inhibitor (Schneider et al., 1980; Stallcup et al., 1979). These similarities suggest that the same transporter catalyzes glutamate flux in both tissues and that the renal system might therefore provide a good model system for studying neuronal glutamate reuptake.

We first demonstrated countertransport with $\mathrm{K}^{+}$for the serotonin transporter of platelet plasma membrane (Rudnick, 1977; Rudnick \& Nelson, 1978). This similarity between glutamate and serotonin transport suggested that other aspects

${ }^{\dagger}$ From the Departments of Pharmacology (P.J.N., G.E.D., and G.R.), Medicine (P.S.A.), and Physiology (P.S.A.), Yale University School of Medicine, New Haven, Connecticut 06510. Received June 3, 1983. P.S.A. and G.R. are established investigators of the American Heart Association. This work was supported by U.S. Public Health Service Grants HL-21217 (to G.R.) and AM-17433 (to P.S.A.). mulation, although $\mathrm{K}^{+}$is not absolutely required for transport. Internal $\mathrm{H}^{+}$inhibits the rate of glutamate accumulation by decreasing this $\mathrm{K}^{+}$stimulation. Conversely, external $\mathrm{K}^{+}$inhibits glutamate influx, and this inhibition can be overcome by lowering the external $\mathrm{pH}$. These results indicate that $\mathrm{H}^{+}$ is cotransported with glutamate and suggest the possibility that, in the absence of internal $\mathrm{K}^{+}, \mathrm{H}^{+}$can also fulfill the requirement for a countertransported ion.

of the transport mechanism might also be similar, especially since both compounds are neurotransmitters. In particular, we were interested in the observation that internal $\mathrm{H}^{+}$substitutes for $\mathrm{K}^{+}$in the serotonin system (Keyes \& Rudnick, 1982). Schneider \& Sacktor (1980) had reported that low $\mathrm{pH}$ also antagonized the stimulation of glutamate transport by internal $\mathrm{K}^{+}$and that glutamate transport was electroneutral both in the presence and absence of internal $\mathrm{K}^{+}$. In the present paper, we have examined in greater detail the effects of internal and external $\mathrm{pH}$ on glutamate transport by renal microvillus membrane vesicles. Our results are consistent with a model in which $\mathrm{Na}^{+}$, glutamate, and $\mathrm{H}^{+}$are cotransported in exchange for $\mathrm{K}^{+}$or $\mathrm{H}^{+}$.

\section{Experimental Procedures}

Preparation of Membrane Vesicles. Microvillus membrane vesicles were isolated from rabbit renal cortex by the magnesium aggregation method described previously (Aronson, 1978). The homogenization and isolation medium consisted of $300 \mathrm{mM}$ mannitol, $10 \mathrm{mM}$ Tris, ${ }^{1}$ and $16 \mathrm{mM}$ Hepes, $\mathrm{pH}$ 7.5. Membranes isolated by this method are purified 14-fold in the luminal membrane marker enzyme, $\gamma$-glutamyltranspeptidase, and are depurified in the basolateral marker, $\mathrm{Na}^{+}, \mathrm{K}^{+}$-ATPase (Aronson, 1978).

Equilibration of Membrane Vesicles. Suspensions of vesicles $(6-10 \mathrm{mg}$ of membrane protein $/ \mathrm{mL}$ by the Lowry assay with BSA as a standard) were diluted at least 10 -fold into media of indicated composition (see figure legends) and incubated for $15 \mathrm{~min}$ at $37^{\circ} \mathrm{C}$. The suspension was then collected by centrifugation at $48000 \mathrm{~g}$ for $20 \mathrm{~min}$ at $4{ }^{\circ} \mathrm{C}$, resuspended in fresh medium of the same composition at 3 times the original protein concentration, and stored at $4{ }^{\circ} \mathrm{C}$ until

'Abbreviations: $\Delta \mathrm{pH}$, the $\mathrm{pH}$ difference across the membrane; Mes, 2-( $N$-morpholino)ethanesulfonic acid; Tris, tris(hydroxymethyl)aminomethane; Hepes, 4-(2-hydroxyethyl)-1-piperazineethanesulfonic acid; BSA, bovine serum albumin; FCCP, carbonyl cyanide $p$-(trifluoromethyl)phenylhydrazone; Mops, 4-morpholinepropanesulfonic acid; ADA, $N$-2-acetamidoiminodiacetic acid. 